\title{
Realistic Spin Glasses Below Eight Dimensions: A Highly Disordered View
}

\author{
C.M. Newman \\ Courant Institute of Mathematical Sciences, New York University, New York, NY 10012 \\ D.L. Stein \\ Departments of Physics and Mathematics, University of Arizona, Tucson, AZ 85721
}

\begin{abstract}
By connecting realistic spin glass models at low temperature to the highly disordered model at zero temperature, we argue that ordinary Edwards-Anderson spin glasses below eight dimensions have at most a single pair of physically relevant pure states at nonzero low temperature. Less likely scenarios that evade this conclusion are also discussed.
\end{abstract}

05.50.+q, 75.10.Nr, 75.50.Lk

Typeset using REVTEX 


\section{INTRODUCTION}

Rigorous [1,2] and non-rigorous [3] analyses ruling out mean-field pictures (for an overview, see [4-6]) of short-ranged finite-dimensional spin glass models leave open the question of whether there is a single pair or infinitely many pure states at low temperature (assuming that spin flip symmetry is indeed broken). The droplet/scaling picture [7-9] asserts the existence of at most a single pair in all dimensions [10,11], but the same conclusion might arise independently of the other predictions of droplet/scaling. On the other hand, if infinitely many pure states occur (for some dimension $d$ and temperature $T$ ), they should do so in accord with the chaotic pairs picture [2, 12,13], in which only a single pair is seen in typical large finite volumes, with the particular pair changing chaotically with volume.

In this paper, we present an analysis that suggests that no multi-pair pictures should occur below eight dimensions. The analysis is based on relating non-zero temperature Gibbs states of Edwards-Anderson(EA) spin glass models [14] to zero-temperature ground states of the highly disordered model [15, 16]. The number of ground state pairs in the highly disordered model is known to be one for $d<8$ and (uncountably) infinite for $d>8$ [15, 17]. The relation is such that the number of ground states in the highly disordered model should serve as an upper bound for the number of (physically relevant) pure states of ordinary EA spin glasses at low but nonzero temperature. We make no claim about the number of ground states of the ordinary models at zero temperature (except by other arguments for $d=2$ [18]).

The idea behind the analysis is as follows. We construct a one-parameter family of EA models, parameterized by $\lambda(\beta)$, where $\beta$ is the inverse temperature, such that $\lambda \rightarrow \infty$ (slowly) as $\beta \rightarrow \infty$ in a way to be specified shortly. The construction is such that for any finite $\beta \geq \beta_{0}$, the model is expected to have the same thermodynamic behavior as the "ordinary" EA nearest-neighbor Ising spin glass in the same dimension corresponding to $\lambda\left(\beta_{0}\right)$, in the sense that if the ordinary EA model is in a low-temperature broken-symmetry spin glass phase at $\beta_{0}$, our model should be also, and the number and organization of pure states in the two models should be identical. At $T=0$ (i.e., $\beta=\infty$ ), our model becomes the highly disordered spin glass [15, 16], whose ground state structure is known [15,17]. So the behaviors of the two models may diverge at $T=0$, i.e., the ground state structure of the highly disordered models may differ from that of the ordinary EA spin glass. But the crucial point is that any $T>0$ Gibbs measure, restricted to any finite volume, of our model becomes supported, as $T \rightarrow 0$, on spin configurations that are ground states of the highly disordered model within that volume.

This results in three possible conclusions, any of which is interesting. The most reasonable follows from the natural expectation that the number of ground states is at least as great as the number of pure states at low temperatures; thus the number of ground states in the highly disordered spin glass should give an upper bound to the number of low-temperature pure states in an ordinary EA model. (There would however be no implication for the number of ground states in the EA model at $T=0$.) Because the highly disordered spin glass has only a single pair of ground states below eight dimensions [15, 17, 19], the conclusion would be that the EA spin glass has no more than a single pair of pure states for all $d<8$ at very low temperature. This conclusion would extend to $d=8$ if it were shown that the highly disordered model has only a single pair of ground states also in that dimension.

We will discuss below why the analysis and this conclusion should apply only to incongruent [20] pure states. Two distinct pure states that are not global flips of each other may be either 
incongruent or regionally congruent. Spin configurations chosen from incongruent pure states have a nonvanishing density of relative domain walls, i.e. couplings satisfied in one but not the other spin configuration; otherwise they are regionally congruent. If incongruence occurs, it should be generated using different sequences of coupling-independent boundary conditions, but to see regional congruence should require choices of boundary conditions that are coupling-dependent.

Two other possibilities, less straightforward but also intriguing, could conceivably occur. One of course is that the supposition that the number of (incongruent) ground states at $T=0$ is no smaller than the number of (incongruent) pure states at low $T>0$ does not hold for this class of models. Another is that there may be no continuity or even monotonicity at all in the number of pure states as a function of temperature in the spin glass — perhaps the most interesting but also least likely of the three possibilities raised here [21].

All three of these conclusions will be discussed in greater detail; we now turn to a discussion of the construction of the class of models to be analyzed.

\section{THE MODEL}

We will study the Edwards-Anderson (EA) Hamiltonian [14] on $\mathbf{Z}^{d}$, the cubic lattice in $d$ dimensions:

$$
\mathcal{H}_{\mathcal{J}}(\sigma)=-\sum_{\langle x, y\rangle} J_{x y} \sigma_{x} \sigma_{y}
$$

where $\mathcal{J}$ denotes a realization of the couplings $J_{x y}$ and where the brackets indicate that the sum is over nearest-neighbor pairs only, with the sites $x, y \in \mathbf{Z}^{d}$. We will take the spins $\sigma_{x}$ to be Ising, i.e., $\sigma_{x}= \pm 1$.

Eq. (11) is the EA Ising Hamiltonian for an infinite-volume spin glass on $\mathbf{Z}^{d}$; we also need to define the EA model on a finite volume, given specified boundary conditions. Let $\Lambda_{L}$ be a cube of side $2 L+1$ centered at the origin; i.e., $\Lambda_{L}=\{-L,-L+1, \cdots, L\}^{d}$. The finite-volume EA Hamiltonian is then just that of Eq. (11) confined to the volume $\Lambda_{L}$, with the spins on the boundary $\partial \Lambda_{L}$ of the cube obeying the specified boundary condition. (The boundary $\partial \Lambda_{L}$ of the volume $\Lambda_{L}$ consists of all sites not in $\Lambda_{L}$ with one nearest neighbor belonging to $\Lambda_{L}$.)

The couplings $J_{x y}$ are quenched, independent, identically distributed random variables; throughout the paper we will assume their common distribution to be symmetric about zero. Most studies use either the Gaussian or $\pm J$ distributions, under the assumption that the qualitative thermodynamic properties in fixed dimension - existence of a phase transition at some $T_{c}(d)$ (whose value, but presumably not its existence, will depend on the nature of the distribution), the presumed broken spin-flip symmetry (i.e., a nonzero EA order parameter $q_{E A}$ ) below $T_{c}$, the number of pure states below $T_{c}$, and so on - will be the same for any "reasonable" coupling distribution. Because this remains an assumption, there is no precise definition of "reasonable", but the expectation is that any distribution that is symmetric about zero and falls off sufficiently quickly for larger coupling magnitudes will all exhibit similar spin glass behavior. Thus, for example, a uniform distribution supported on $[-1,1]$ is expected to have the same basic thermodynamic properties as the $\pm J$ or Gaussian spin glasses. We proceed using this assumption (or weakened versions of it).

We depart from previous studies in parametrizing the coupling distribution through the variable $\lambda(\beta)$, with $\lambda \rightarrow \infty$ as $\beta \rightarrow \infty$. The couplings $J_{x y}$ are defined through the relation (cf. [15]) 


$$
J_{x y}^{\lambda(\beta)}=\epsilon_{x y} c_{\lambda(\beta)} e^{-\lambda(\beta) K_{x y}}
$$

where $c_{\lambda(\beta)}$ is chosen to ensure that the model has a sensible thermodynamic limit (i.e., a finite energy/spin) when $\beta \rightarrow \infty$, and $\epsilon_{x y}$ and $K_{x y}$ are two sets of independent, identically distributed (i.i.d.) random variables. Each variable $\epsilon_{x y}$ takes on one of the two values \pm 1 with equal probability, and the $K_{x y}$ can be chosen from any continuous distribution (e.g. uniformly from [0,1]) so that the distribution of the $J_{x y}$ 's is "reasonable", as discussed above. Two examples will be given shortly.

Our general approach is to start with a "typical" EA model at $\lambda\left(\beta_{0}\right)=1$, and then embed this within a one-parameter family of models that has a known ground state structure (corresponding to $\lambda(\infty)=\infty$ ). Let us denote by $J_{0}$ a given random coupling at the origin connecting to a specified nearest-neighbor site. By Eq. (2), for a given realization of the random variables $\epsilon$ and $K$, we have $J_{0}=\epsilon_{0}\left|J_{0}\right|=\epsilon_{0} c_{1} e^{-K_{0}}$ ( $c_{1}$ can be taken equal to 1 ). After a rescaling of the temperature, we have

$$
J_{0}^{\lambda}=\epsilon_{0} c_{\lambda}\left|J_{0}^{\lambda}\right|=\epsilon_{0} c_{\lambda} e^{-\lambda K_{0}} .
$$

We will see that a choice of $c_{\lambda} \sim 1 / \overline{\left|J_{0}\right|^{\lambda}}$, where an overbar denotes an average over coupling realizations, will ensure a sensible thermodynamics for the model in the limit of zero temperature (providing $\lambda$ increases slowly as $\beta \rightarrow \infty$ ).

Note that when $\lambda=0$ the model becomes the $\pm J$ model for any starting choice of coupling distribution. Of more relevance is the opposite limit: as $\lambda \rightarrow \infty$, all initial coupling distributions merge into the highly disordered model discussed in [15-17]. In this limit the coupling distribution is infinitely "stretched" nonlinearly, so that every coupling magnitude occurs on its own scale. A full analysis of the ground state structure of this model, and the transition in ground state pair multiplicity, is given in [15, 17]. A quantum version of the highly disordered limit has been used to study the random quantum Ising model in a transverse field [22].

So given a wide range of choices for the distribution of the $K$ 's, the spin glass model described here has a "reasonable" coupling distribution for any $\beta<\infty$; we will in fact work with coupling distributions that have a large-magnitude cutoff at finite values (depending on $\beta$ ), which guarantees this. As $\beta$ increases, the distribution becomes increasingly stretched, but retains its finite largemagnitude cutoff for any $\beta<\infty$, so that any of these models retains the qualitative thermodynamic behavior of the ordinary EA model.

As we will see, the dependence of $\lambda$ on $\beta$ can be chosen (with $\lambda$ increasing sufficiently slowly) so that if an ordinary EA model, corresponding to some finite $\lambda$ (e.g., $\lambda\left(\beta_{0}\right)=1$ ), is in a lowtemperature spin glass phase, then so is the model described above with running $\lambda$. We will further see that if $\lambda$ increases slowly enough with $\beta$ (depending on the choice of distribution for $K)$, then the Gibbs measures in the limit $\beta \rightarrow \infty$ are supported only on spin configurations that are ground states of the highly disordered model. That is natural since taking $\lambda(\beta) \rightarrow \infty$ slowly enough is roughly equivalent to first taking the $\beta \rightarrow \infty(T \rightarrow 0)$ limit and then the $\lambda \rightarrow \infty$ limit.

\section{A. Two examples}

In this section we give two examples of coupling realizations that can be used in subsequent analyses. As already noted, the precise form of the distribution is unimportant as long as the requirements listed earlier are met; all such models should exhibit the same positive-temperature 
behavior and will merge at zero temperature. The first example starts with a flat distribution for $J_{x y}$ in the interval $[-1,1]$ at $\lambda\left(\beta_{0}\right)=1$. We need to determine the distribution for $K$ necessary to recover this flat distribution for $J$. Let

$$
\mathcal{U}^{\lambda}=e^{-\lambda K}
$$

with $\mathcal{U}^{1}=e^{-K}$ having a flat distribution in $[0,1]$.

It follows that $K$ is taken from an exponential distribution on $(0, \infty)$, with $P(K) d K=$ $e^{-K} d K$. Now let $J_{x y}^{\lambda}=\epsilon_{x y} c_{\lambda} \mathcal{U}_{x y}^{\lambda}$; then $M=\left|J_{x y}^{\lambda}\right|$ has the distribution

$$
P(M) d M= \begin{cases}\lambda^{-1}\left(M / c_{\lambda}\right)^{1 / \lambda} M^{-1} d M, & \text { if } 0<M<c_{\lambda}, \\ 0, & \text { otherwise } .\end{cases}
$$

It remains to choose $c_{\lambda}$. The procedure for doing this will be discussed in Sec. IVB; we here give the result, which is $c_{\lambda}=\lambda$, so that $J_{x y}^{\lambda}=\lambda \epsilon_{x y} \mathcal{U}_{x y}^{\lambda}$ and

$$
P(M) d M= \begin{cases}(1 / \lambda)[M / \lambda]^{1 / \lambda} M^{-1} d M, & \text { if } 0<M<\lambda, \\ 0, & \text { otherwise } .\end{cases}
$$

The second example starts with a uniform distribution for $K$ on [0,1]. Again

$$
J_{x y}^{\lambda}=\lambda \epsilon_{x y} e^{-\lambda K_{x y}}
$$

where we have already chosen $c_{\lambda}=\lambda$, but the distribution of $M=\left|J_{x y}^{\lambda}\right|$ is now given by

$$
P(M) d M= \begin{cases}(1 / \lambda) M^{-1} d M, & \text { if } \lambda e^{-\lambda}<M<\lambda, \\ 0, & \text { otherwise }\end{cases}
$$

The coupling distribution for this case is graphed in Fig. 1. 


\section{FIGURES}

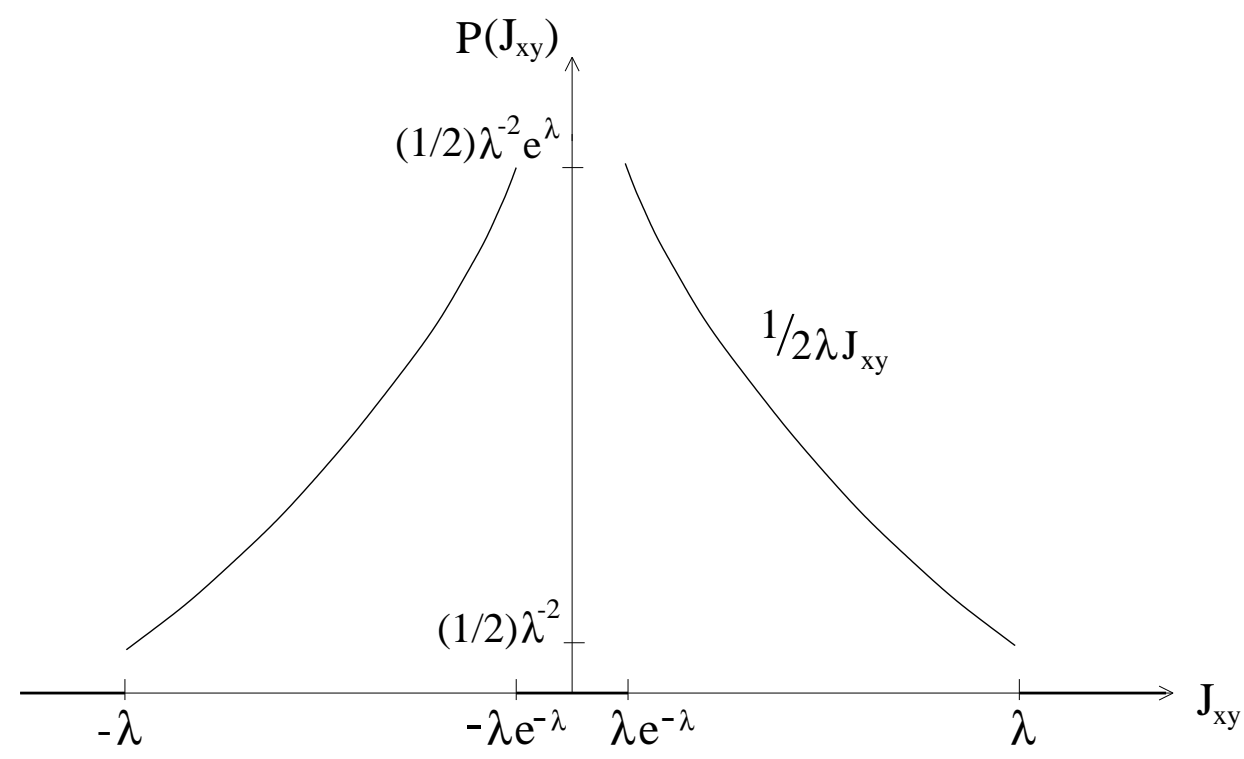

FIG. 1. Sketch of the coupling density $P\left(J_{x y}\right) d J_{x y}$ from Eq. (8) for fixed $\lambda<\infty$, corresponding to a uniform distribution of $K_{x y}$ on $[0,1]$.

It is important to note that as $\lambda \rightarrow \infty$, the distributions of $J_{x y}^{\lambda}$ in the two examples approach each other; this is true in general. Both of these distributions have a temperature-dependent cutoff at large coupling magnitudes. While distributions with cutoffs are chosen for convenience, one could also choose a Gaussian distribution for $J_{x y}$ at $\lambda\left(\beta_{0}\right)=1$. Because all of these distributions are expected to give the same thermodynamic behavior, however, we will henceforth use the simplest of these, given by Eqs. (7) and (8).

\section{PURE STATE STRUCTURE WITHIN THE METASTATE}

We will study this class of models and will show that (for slow enough increase of $\lambda$ with $\beta$ ), as $\beta \rightarrow \infty$, the Gibbs measures (restricted to arbitrary fixed volumes $\Lambda_{L}$ ) become supported on the ground states of the highly disordered spin glass. We require that $c_{\lambda}$ be chosen so that the model will be sensible in the zero-temperature limit, in that that the energy density converges to a finite nonzero value, and further that the choice of $\lambda(\beta)$ be made in such a way that, for each $\beta>\beta_{0}$, the model always remains within the low temperature broken-spin-flip-symmetric spin glass phase (assuming the corresponding ordinary EA model at $\beta_{0}$ is in such a phase).

If these requirements can be met, then the most natural conclusion is that in any dimension $d$, the number of ground states of the highly disordered model provides an upper bound to the number of pure states observed at low (but nonzero) temperature in the ordinary EA spin glass. (We emphasize again, though, that other conclusions remain possible, though perhaps less plausible; these will be discussed in Sec. VI.)

As we have emphasized in earlier papers, if there are multiple pure states, the interesting, and physically relevant, situation is the occurrence of incongruent states. Regional congruence is of mathematical interest, but to see it would require a choice of boundary conditions carefully 
conditioned on the coupling realization $\mathcal{J}$. It is not currently known how to choose such b.c.'s in spin glasses. Numerical treatments that look for multiple pure states implicitly search for incongruent ones. (It is interesting to note that recent numerical studies [24]26], some employing coupling-dependent bulk terms in the Hamiltonian, have suggested the possibility of observation of regionally congruent states; but see also [27] for a different interpretation of the numerical data. However, recent work indicates that the energetics of the interfaces found in these studies are inconsistent with regionally congruent pure or ground states [28].)

We expect our analysis to hold for the number of (incongruent) pure states within any of the coupling-independent boundary condition metastates. The concept of metastate was introduced and discussed in Refs. [2] and [13], and shown to be equivalent to an earlier (but somewhat different) construct in Ref. [23].

A metastate is a measure on (infinite-volume) Gibbs states at fixed temperature that is constructed via an infinite sequence of volumes $\Lambda_{L}$, with specified boundary conditions on each $\partial \Lambda_{L}$ chosen in a coupling-independent manner. Roughly speaking, the metastate provides the probability (for varying large $L$ ) of various Gibbs states appearing within $\Lambda_{L}$. The equivalence of metastates constructed with certain different coupling-independent boundary conditions was shown in Ref. [3].

If there are infinitely many (incongruent) pure states, a metastate should be dispersed over them, giving their relative likelihood of appearance in typical large volumes. If there is no incongruence, the metastate should be unique and supported on a single pure state pair, and that pair will appear in most (i.e., a fraction one) of the $\Lambda_{L}$ 's. Regionally congruent states, if present, would be "invisible" in the metastate, i.e., would appear in a vanishing fraction of all the $\Lambda_{L}$ 's. Hence, even if regional congruence existed, there would still be two special or "preferred" pure states that would be seen in a typical $\Lambda_{L}$ with $L$ large.

An example of regional congruence is afforded by interface states in ferromagnets, which can only be seen with carefully chosen boundary conditions, such as those of Dobrushin [29]. These are b.c.'s in which the boundary spins above the "equator" (a plane or hyperplane parallel to two opposing faces of $\Lambda_{L}$ and cutting it essentially in half by passing at a finite height above or below the origin) are chosen to be plus and the boundary spins below the equator are minus. Here the special pair of states consists of the uniformly magnetized up and down states, which are seen in large volumes with random boundary conditions as well, of course, as with periodic or free b.c.'s. (They would also be seen even with antiperiodic b.c.'s, since the interface would pass through any fixed finite region only in a vanishing fraction of volumes.) An important difference with the spin glass case is that in the latter there is no known procedure for obtaining boundary conditions that would "see" any analogous regionally congruent states.

In the current context, one needs to specify which Gibbs measures are being examined at $T>0$. The procedure in which one chooses coupling-independent boundary conditions and then varies the temperature is well-defined when one is studying properties of the metastate as a whole. As noted, this will provide information on the number of incongruent pure states (in the metastate) at a given temperature. If one wants to push further in order to study the possible existence of regionally congruent pure states, then one needs to pick out these Gibbs states through a choice of coupling-dependent boundary conditions. In order to take the $\beta \rightarrow \infty$ limit, one would then have to change these coupling-dependent boundary conditions in some unknown way; in this case, the procedure of taking the zero-temperature limit is a priori not well-defined in general. For spin glasses, the only well-defined procedures known at this time use coupling-independent b.c.'s, as 
$T \rightarrow 0$.

The consequence is that this argument is best able to provide information on the number of incongruent states in the spin glass metastate at various positive temperatures, but that it is harder to draw conclusions on the possible existence or nonexistence of regionally congruent states. These latter are the interface states that are "invisible" in coupling-independent metastates, and in any case, as we have argued in earlier papers [2, 3, 18], are unlikely to be physically relevant even if they exist, since boundary conditions used in (either numerical simulations or) laboratory experiments on spin glasses are coupling-independent - i.e., they are not tailored to the microscopic disorder configuration. (For other results and discussion concerning the distinction between couplingdependent and independent boundary conditions, see [30,31].)

\section{ANALYSIS}

We now analyze the behavior of our class of models both at large finite $\lambda$ (i.e., at low temperature) and in the $\lambda \rightarrow \infty$ limit. As noted above, for specificity we will study the behavior of the model with coupling distribution given by Eqs. (7) and (8).

Our argument relies on answering three questions (in the affirmative). For a given model (i.e., specified coupling distribution) can one choose a $c_{\lambda}$ and a $\lambda(\beta)$ (independently of particular coupling realization) so that:

1) the model has a thermodynamically sensible scaling limit as $\beta \rightarrow \infty$, in that the energy per spin converges to some $C$ as $\beta \rightarrow \infty$, with $0<C<\infty$;

2) $\lambda(\beta)$ scales in such a way to ensure that, if the model is in its low temperature thermodynamic phase originally, i.e., at $\lambda\left(\beta_{0}\right)=1$, the model at rescaled (lower) temperature remains in that same phase;

3) $\lambda(\beta)$ increases slowly enough with $\beta$ to ensure that any Gibbs state at positive temperature is increasingly supported, as temperature is lowered, on spin configurations that are ground states of the highly disordered model?

In the following three subsections, we provide an analysis that answers these questions in the affirmative, and shows how such a $c_{\lambda}$ and $\lambda(\beta)$ can be chosen.

\section{A. Effective temperature and couplings}

In this subsection we present a simple transformation on the (inverse) temperature and the couplings that enables us to map the class of models under study onto more familiar ones. In the absence of a scaling factor $c_{\lambda}$, the Gibbs weighting factor at inverse temperature $\beta^{\prime}$ is

$$
\exp \left[-\beta^{\prime} \sum_{\langle x y\rangle} \epsilon_{x y} e^{-\lambda K_{x y}} \sigma_{x} \sigma_{y}\right] \text {. }
$$

We transform the temperature and couplings using

$$
\beta^{\prime} \epsilon_{x y} e^{-\lambda(\beta) K_{x y}}=\beta J_{x y}^{\lambda(\beta)}
$$

where $\beta^{\prime}=\beta c_{\lambda(\beta)}$ and 


$$
J_{x y}^{\lambda(\beta)}=\epsilon_{x y} c_{\lambda(\beta)} e^{-\lambda(\beta) K_{x y}},
$$

so at a given $\beta^{\prime}$ the model maps onto an equivalent one at effective temperature $\beta$ and with effective couplings $J_{x y}^{\lambda(\beta)}$. Although simple, this rewriting of the Gibbs factor provides a natural separation of the various factors that allows us to choose the prefactor $c_{\lambda}$ in a coupling-independent way, so that the energy density has a sensible $\beta \rightarrow \infty$ limit.

To achieve the correct scaling limit, we need to choose $c_{\lambda(\beta)}$ so that it scales (with $\beta$ or $\beta^{\prime}$ ) as the inverse of (minus) the energy density $e\left(\beta^{\prime}, \lambda\right)$ of the model with Gibbs factor (9). This energy density $e\left(\beta^{\prime}, \lambda\right)$ is the same for all (infinite-volume) Gibbs states and for almost all realizations of the couplings (under the assumption that $\overline{\left|J_{x y}^{\lambda}\right|}<\infty$ ) and so equals its disorder average $\overline{e\left(\beta^{\prime}, \lambda\right)}$. Since $\beta^{\prime}$ (and $\beta$ ) will be chosen to scale to infinity rapidly as functions of $\lambda$, we will choose $c_{\lambda}$ to scale like the inverse of (minus) $\overline{e(\infty, \lambda)}$, the ground state energy density, which in turn scales like (minus) $\overline{\left|J_{x y}^{\lambda}\right|}$ as $\lambda \rightarrow \infty$. To justify this choice of $c_{\lambda}$, we need to compute $e\left(\beta^{\prime}, \lambda\right)$ in the low-temperature limit. This is done in the next section.

\section{B. Thermodynamic behavior of the zero temperature limit}

In this subsection we estimate the disorder-averaged energy per spin $\overline{e\left(\beta^{\prime}, \lambda\right)}$. Although $\beta^{\prime}$ and $\lambda$ will later be taken to depend on each other, for the purposes of this section we treat them as independent variables. One of the purposes of this calculation is to provide information on how slowly $\lambda$ needs to vary with $\beta^{\prime}$ (and hence with $\beta$ ) in order to have sensible thermodynamics in the zero temperature limit. In Sec. IVD we will study how $\lambda$ needs to vary with $\beta$ in order for pure states at positive temperature to be supported on ground states of the highly disordered model. We will see that if $\lambda$ grows slowly with $\beta^{\prime}$ and $\beta$, these two calculations result in mutually compatible ranges for allowed scaling behaviors.

We now proceed to show that the energy per spin, at large $\beta$, can be computed as a disorder average over a single (arbitrary) coupling $J_{0}^{\lambda}=e^{-\lambda K_{0}}$, which as before denotes the coupling (in $\mathcal{J}$ ) connecting the spin at the origin with one of its nearest neighbors. Let $P_{\beta^{\prime}, \lambda}$ denote the probability (within a Gibbs measure at fixed $\beta^{\prime}$ ). Then, in dimension $d$,

$$
-\overline{e\left(\beta^{\prime}, \lambda\right)}=d \overline{\left(P_{\beta^{\prime}, \lambda}\left(J_{0}^{\lambda} \text { is satisfied }\right)-P_{\beta^{\prime}, \lambda}\left(J_{0}^{\lambda} \text { is unsatisfied }\right)\right)\left|J_{0}^{\lambda}\right|} .
$$

At large $\lambda$ (and larger $\beta^{\prime}$ ), the main contribution to the energy density arises from couplings corresponding to the smallest $K_{x y}$ 's, and their probability of being satisfied approaches one. Moreover,

$$
0=-\overline{e(0, \lambda)} \leq-\overline{e\left(\beta^{\prime}, \lambda\right)} \leq-\overline{e(\infty, \lambda)}<\overline{d \mid \overline{J_{0}^{\lambda} \mid}},
$$

where the last inequality is because not every coupling is satisfied at zero temperature. So it will be sufficient for our purposes to derive a lower bound on $-\overline{e\left(\beta^{\prime}, \lambda\right)}$ that approaches $d \overline{\left|J_{0}^{\lambda}\right|}$ as $\beta \rightarrow \infty$. In arriving at a lower bound, the following general inequality will be useful:

$$
\begin{aligned}
P_{\beta^{\prime}, \lambda}\left(J_{0}^{\lambda} \text { is satisfied }\right)-P_{\beta^{\prime}, \lambda}\left(J_{0}^{\lambda} \text { is unsatisfied }\right) & \\
& =1-2 P_{\beta^{\prime}, \lambda}\left(J_{0}^{\lambda} \text { is unsatisfied }\right) \\
& \geq 1-2 \frac{P_{\beta^{\prime}, \lambda}\left(J_{0}^{\lambda} \text { is unsatisfied }\right)}{P_{\beta^{\prime}, \lambda}\left(J_{0}^{\lambda} \text { is satisfied }\right)} .
\end{aligned}
$$


We use the coupling magnitude distribution Eq. (8), which arises from the flat distribution for $K$ on the unit interval $[0,1]$. Because most of the contribution to the energy density comes from couplings corresponding to small $K_{x y}$ 's, we break up the calculation into three cases according to the realization of $K_{0}$ and its $2(2 d-1)$ adjacent couplings:

Case I: $K_{0} \geq \delta$, where $\delta$ is fixed and $0<\delta \ll 1$;

Case II: $K_{0}<\delta$, and one or more adjacent couplings have magnitudes corresponding to $K \leq b_{0} \delta$, where $b_{0}$ is a constant with $1 / \delta>b_{0}>1$;

Case III: $K_{0}<\delta$, and all adjacent couplings have magnitudes corresponding to $K>b_{0} \delta$.

If we denote by $e_{I}$ the contribution to (minus $1 / d$ times) the energy density from coupling realizations corresponding to Case I, and similarly for the other cases, then trivially

$$
-(1 / d) \overline{e\left(\beta^{\prime}, \lambda\right)}=e_{I}+e_{I I}+e_{I I I}
$$

We now study the three cases separately, denoting by $\mathcal{I}_{I}, \mathcal{I}_{I I}$ and $\mathcal{I}_{I I I}$ the indicator functions that equal 1 (otherwise 0 ) only for those coupling realizations satisfying respectively the requirements of the three different cases.

Case I: Here we have

$$
e_{I} \leq \overline{\mathcal{I}_{I}\left|J_{0}^{\lambda}\right|}=\int_{\delta}^{1} e^{-\lambda K} d K=O\left((1 / \lambda) e^{-\lambda \delta}\right)
$$

as $\lambda \rightarrow \infty$.

Case II: The calculation here is similar; we have

$$
e_{I I} \leq \overline{\mathcal{I}_{I I}\left|J_{0}^{\lambda}\right|} \leq 2(2 d-1) b_{0} \delta \int_{0}^{\delta} e^{-\lambda K} d K=O(\delta) \int_{0}^{\delta} e^{-\lambda K} d K
$$

as $\lambda \rightarrow \infty$.

Case III: This case is more involved. There are four possible configurations for the two spins coupled through $J_{0}^{\lambda}$; two of these correspond to $J_{0}^{\lambda}$ satisfied and two to $J_{0}^{\lambda}$ unsatisfied. Consider the ratio $\frac{P_{\beta^{\prime}, \lambda}\left(J_{0}^{\lambda} \text { is unsatisfied }\right)}{P_{\beta^{\prime}, \lambda}\left(J_{0}^{\lambda} \text { is satisfied }\right)}$ that appears in Eq. (14). This ratio is maximized by the following "worst case" scenario: in changing a satisfied configuration (for $J_{0}^{\lambda}$ ) to an unsatisfied one, all of the $2 d-1$ adjacent couplings touching the flipped spin change from unsatisfied to satisfied. This case maximizes the cost in the Gibbs factor for the change in spin configurations. It follows that under the requirements of case III,

$$
\frac{P_{\beta^{\prime}, \lambda}\left(J_{0}^{\lambda} \text { is unsatisfied }\right)}{P_{\beta^{\prime}, \lambda}\left(J_{0}^{\lambda} \text { is satisfied }\right)} \leq \exp \left[-2 \beta^{\prime} e^{-\lambda \delta+(2 d-1) 2 \beta^{\prime} e^{-b_{0} \lambda \delta}}\right] .
$$

Therefore

$$
\begin{aligned}
e_{I I I} & =\frac{\overline{\mathcal{I}_{I I I}\left|J_{0}^{\lambda}\right|\left(1-2 P_{\beta^{\prime}, \lambda}\left(J_{0}^{\lambda} \text { is unsatisfied }\right)\right)}}{} \\
& =[1-O(\delta)]\left(1-O\left[e^{-2 \beta^{\prime} e^{-\lambda \delta}+(2 d-1) 2 \beta^{\prime} e^{-b_{0} \lambda \delta}}\right]\right) \int_{0}^{\delta} e^{-\lambda K} d K
\end{aligned}
$$

as $\lambda \rightarrow \infty$.

The behavior of the factor $e^{-2 \beta^{\prime} e^{-\lambda \delta}+(2 d-1) 2 \beta^{\prime} e^{-b_{0} \lambda \delta}}$ that appears in Eq. (19) as $\beta^{\prime} \rightarrow \infty$ and $\lambda \rightarrow \infty$ is sensitive to the dependence of these two parameters on each other. We have 


$$
e^{-2 \beta^{\prime} e^{-\lambda \delta}+(2 d-1) 2 \beta^{\prime} e^{-b_{0} \lambda \delta}}=e^{-2 \beta^{\prime} e^{-\lambda \delta}\left(1-(2 d-1) e^{-\left(b_{0}-1\right) \lambda \delta}\right)} \sim e^{-2 \beta^{\prime} e^{-\lambda \delta}} \text { as } \lambda \rightarrow \infty
$$

regardless of the detailed behavior of $\lambda(\beta)$. Furthermore, $\beta^{\prime} e^{-\lambda \delta} \rightarrow \infty$ for any $\delta$, if $\lambda \rightarrow \infty$ slower than $\log \left(\beta^{\prime}\right)$ (i.e., if $\lambda / \log \left(\beta^{\prime}\right) \rightarrow 0$ ). We will therefore require (for this particular coupling distribution, but the calculation is similar for others) that

$$
\lambda=o[\log (\beta)] \text { as } \beta \rightarrow \infty,
$$

which, as we shall see, will also guarantee that $\lambda=o\left[\log \left(\beta^{\prime}\right)\right]$.

Returning to the comparison of the energy densities (as $\beta^{\prime} \rightarrow \infty$ ) for the three cases, we see that $e_{I}$ is reduced from $e_{I I I}$ by a factor of order $e^{-\lambda \delta}$, and $e_{I I}$ is reduced from $e_{I I I}$ by a factor of order $\delta$. We therefore find that

$$
-\overline{e\left(\beta^{\prime}, \lambda\right)}=d[1 \pm O(\delta)] \int_{0}^{1} e^{-\lambda K} d K
$$

so that in the joint limit $\beta^{\prime} \rightarrow \infty, \lambda \rightarrow \infty$ (and with the condition $\lambda=o\left[\log \left(\beta^{\prime}\right)\right]$ satisfied for $K$ uniformly distributed on $[0,1]$ ), since $\delta$ can be chosen arbitrarily small, it follows that

$$
-\overline{e\left(\beta^{\prime}, \lambda\right)} /\left(d \overline{\left|J_{0}^{\lambda}\right|}\right) \rightarrow 1 \text { as } \beta^{\prime} \rightarrow \infty
$$

Therefore, if $K \in[0,1]$ uniformly, we have

$$
-\overline{e\left(\beta^{\prime}, \lambda\right)} \sim \overline{\left|J_{0}^{\lambda}\right|}=\overline{e^{-\lambda K_{0}}}=\int_{0}^{1} e^{-\lambda K} d K \sim 1 / \lambda \text { as } \lambda \rightarrow \infty,
$$

and we set $c_{\lambda}=\lambda$ for this distribution, as in Eq. (7). Thus $\beta^{\prime}=\beta c_{\lambda}=\beta \lambda$ and (21) will indeed guarantee $\lambda=o\left[\log \left(\beta^{\prime}\right)\right]$. A similar calculation for the distribution of Eq. (5), discussed in Sec. IIA, results in $c_{\lambda} \sim \lambda+1$, and so we may choose $c_{\lambda}=\lambda$. More generally, one chooses $c_{\lambda} \sim 1 /\left|J_{0}^{\lambda}\right|$, as discussed in Sec. II, to obtain a finite, nonzero energy density as temperature goes to zero.

\section{Comparison to phase behavior of ordinary EA models}

We have shown that the first of our requirements, that the thermodynamics of our class of models behave properly in the zero temperature limit, can be met, and have shown how to compute the energy density at low temperatures. We turn now to our second requirement, namely that the model remain in the low temperature spin glass phase as $T$ is lowered from a starting temperature already within the spin glass phase for the $\lambda\left(\beta_{0}\right)$ model. Of course, the low-temperature behavior of the ordinary EA spin glass in dimensions three and higher is not well understood, and it could conceivably be the case that in some or all dimensions between three and eight it undergoes a succession of phase transitions (either at discrete temperatures or continuously), or has no phase transition at positive temperature at all (i.e., remains paramagnetic down to zero temperature), or has other, perhaps more exotic, behavior. Our only goal here is to show that our class of models behaves similarly to the ordinary EA spin glass at least for very low, nonzero temperatures. To keep matters simple, we'll assume that for some range of $d$, the ordinary EA spin glass undergoes a phase transition at some $T_{c}(d)>0$, such that below that temperature there is a spin glass 
phase with broken spin flip symmetry, and with the number (and organization) of pure states not depending on $T$, for $0<T<T_{c}(d)$.

That our class of models should behave similarly (at $\beta<\infty)$ to ordinary EA models, in terms of numbers and organization of pure states as $\beta$ changes, is not immediately obvious because the couplings in our models are temperature-dependent. It does seem reasonable to expect though that, so long as the couplings depend weakly on $\beta$, our models should behave similarly to more conventional ones. However, we can improve on this expectation and show that in fact this follows from a natural universality hypothesis.

The universality we have in mind is that the above assumption about ordinary EA spin glasses is valid separately for each fixed finite $\lambda$. Thus there will be a critical inverse temperature $\beta_{c}(\lambda)<$ $\infty$ for the Hamiltonian Eq. (1) with couplings $J_{x y}=\epsilon_{x y} c_{\lambda} e^{-\lambda K_{x y}}$. In order to satisfy our second requirement, $\lambda$ has to grow slowly enough with $\beta$ (or equivalently, $\beta$ has to grow rapidly enough with $\lambda$ ) so that for $\beta \geq \beta_{0}$,

$$
\beta(\lambda)>\beta_{c}(\lambda)
$$

So, for example, if $\lambda\left(\beta_{0}\right)=1$ and the corresponding spin glass model is in its low $T$ spin glass phase, the inequality (25) will guarantee that the sequence of models with running $\lambda>1$ remain within their corresponding low $T$ spin glass phase. The above inequality can always be satisfied consistently with the other conditions 1) and 3); all that needs to be done is to choose $\lambda$ to grow sufficiently slowly with $\beta$. The remaining question will be whether the constraints imposed by conditions 1) and 3) are compatible; we reserve that for the following section.

\section{Ground state behavior of zero temperature limit}

We now turn to a central question in the analysis of our models, which is how slowly $\lambda$ must vary with $\beta$ in order that condition 3) above is satisfied, i.e., whether the Gibbs states of our models at positive temperature are increasingly supported, as temperature is lowered, on spin configurations that are ground states of the highly disordered model, and whether this constraint is compatible with condition 1).

Consider again a fixed (arbitrary) volume $\Lambda_{L}$ centered at the origin, with any fixed boundary condition chosen independently of the coupling realization. Let $\Delta E(\lambda)$ denote the energy difference between the lowest-energy state in $\Lambda_{L}$ at given $\lambda$ and the first excited state. Then condition 3 ) is satisfied if $\beta \Delta E(\lambda(\beta)) \rightarrow \infty$ as $\beta \rightarrow \infty$, because the lowest-energy state goes to the ground state of the highly disordered model (for that b.c., in that volume) as $\lambda \rightarrow \infty$.

We again study this question in the context of a particular model, namely where $K$ is chosen

uniformly from the interval $[0,1]$. We have here that $J_{x y}^{\lambda} \sim \lambda \epsilon_{x y} e^{-\lambda K_{x y}}$. As $\lambda$ gets large for fixed $L$, the first excited state corresponds to the (multi-)spin flip that changes only the smallest magnitude coupling in the (wired b.c.) invasion tree (see Refs. [15, 17]]) from satisfied to unsatisfied. But for the distribution chosen, the magnitude of this coupling is larger than $\lambda \exp [-\lambda]$, so that at any $\lambda, \Delta E(\lambda)>\lambda e^{-\lambda}$. Therefore,

$$
\beta \Delta E(\lambda) \geq \beta \lambda e^{-\lambda},
$$

so if $\lambda$ grows as $\log (\beta)$ or slower, condition 3 ) is satisfied (for this particular distribution of $K$ ). 
Condition 1) requires a slightly stronger constraint, given by (21), which is certainly compatible with the constraint given above. It is gratifying that both conditions require $\lambda$ to grow slowly with $\beta$, as initially anticipated. It might be that condition 2) would require $\lambda$ to grow even more slowly with $\beta$, but in any event poses no conflicts with the other constraints.

The procedure given here can be adapted for other distributions, but it is already sufficient that an explicit example can be constructed of a model with all of the desired properties listed earlier.

\section{DISORDERED FERROMAGNETS}

The basic argument of this paper is that for the models with temperature dependent couplings as in Eq. (2) (and with properly chosen $c_{\lambda}$ and $\lambda(\beta)$ ), the absence of more than a single pair of ground states at $\beta=\infty$ for $d<8$ is evidence for no more than two pure states in ordinary EA spin glass models with fixed couplings at very low temperatures.

A potential flaw in this line of reasoning seems to arise in the case of disordered ferromagnets, corresponding to the elimination of the random signs $\epsilon_{x y}$ in Eq. (2). After all, highly disordered ferromagnets also have only a single pair of ground states for $d<8$ [15, 17], but ordinary disordered ferromagnets are expected to have multiple pure states for $d \geq 4$ [32] - namely, the interface states [33, 34] obtained by using Dobrushin boundary conditions. Further, this expectation is strongly supported by the rigorous results of Bovier and Külske concerning the existence for $d \geq 4$ of such interface states in SOS models [35]. Indeed, the existence of such interface states in ferromagnets motivated Bovier and Fröhlich to argue that there are more spin glass pure states in $d \geq 4$ than $d=3$ (see Sec. 6.4 of [36]). So we need to ask why our arguments, when applied to disordered ferromagnets, do not lead to a contradiction for $4 \leq d<8$.

In fact, they do not, for at least two reasons. One of them, already discussed above, is the lack of appearance of interface states in the metastate (with coupling independent boundary conditions). We have not argued, even for spin glasses, that there are absolutely no more than two pure states for $d<8$, but only that not more than two appear in the metastate (and that these are the physically relevant ones). For both ordinary EA spin glasses in $d<8$ and ordinary disordered ferromagnets in all $d$, the metastate with free or periodic (or random) b.c.'s should exhibit no more than a single pair of pure states. Indeed, in the highly disordered ferromagnet even for $d>8$, the free or periodic (but not random) b.c. metastate is supported only on the single pair of homogeneously magnetized ground states [15, [7]].

But one might object that in the ferromagnet setting, unlike the spin glass case, one does know how to choose b.c.'s, namely of the Dobrushin type, so that one can obtain Gibbs states with interfaces in the thermodynamic limit and metastates supported on them. For example, by taking a mixed b.c. with the Dobrushin "equator" between the plus and minus parts of the boundary occuring at a variety of heights with various weights, one could obtain a metastate supported on many different pure interface states. So, wouldn't the fact that for $4 \leq d<8$, the zero temperature limit of the metastate could not be supported on more than two ground states, still contradict our reasoning that the number of ground states in the metastate at zero temperature is an upper bound for the number of pure states at very low temperature?

Here is where the second reason for a distinction between ferromagnets and spin glasses comes into play. It concerns the universality hypothesis of our argument, that for any fixed $\lambda<\infty$, the low temperature phase structure of a spin glass should be qualitatively the same, regardless of the value of $\lambda$. For spin glasses, this seems a perfectly plausible working hypothesis; indeed, if 
this were not so, then, e.g., a Gaussian distribution could have a different low $T$ thermodynamic structure, in terms of pure state multiplicity, than a uniform distribution on $[-J, J]$, or a $\pm J$ spin glass. But this is not likely; it is expected that only non-universal features such as $T_{c}(d)$ depend on the coupling distribution (assuming that it is symmetric about zero and without slowly-decaying tails).

But this is not so for disordered ferromagnets, where there are no energy cancellations along an interface - unlike in spin glasses. This may well lead to a greater sensitivity of interface stability to the strength of the disorder. It seems quite plausible, as suggested to us by Bovier and Külske, that for some dimensions $d \geq 4$, the usual interface states obtained through normal Dobrushin b.c.'s could perhaps disappear for $\lambda$ above some critical value $\lambda_{c}<\infty$, even for arbitrarily low temperature. That is, for $\lambda<\lambda_{c}$, the interface would be flat for both $T=0$ and small $T>0$, but for $\lambda>\lambda_{c}$, the interface would be rough for any $T>0$ (and perhaps also for $T=0$ ).

Our analysis suggests that this is indeed so for $d<8$, so that there would be only two pure states (even for the mixed Dobrushin b.c. metastate described above) for ordinary disordered ferromagnets at very low temperatures, providing the disorder is sufficiently (but not infinitely) strong. For $4 \leq d<8$, this could be a consequence of the strong disorder either completely destabilizing the interface states so that they are entirely absent, or else of partially destabilizing them so that finding them would require Dobrushin type b.c.'s, but with a coupling-dependent equator (at a nonconstant height).

\section{DISCUSSION}

In this paper we have constructed a class of nearest-neighbor spin glass models with (unusual) coupling distributions depending on a disorder strength parameter $\lambda$ that itself is temperature dependent. These models are designed to have the property that their thermodynamic equilibrium properties at low temperature $T$ should be the same as for models with more familiar distributions (e.g., $\pm J$ or Gaussian), but they have the rare advantage that their ground state structure is known. The basic assumption of this paper is that a nearest-neighbor Ising spin glass model with any "reasonable" coupling distribution - i.e., symmetric about zero, and with small or zero weight on very large coupling magnitudes - will display qualitatively equivalent thermodynamics at fixed $d$ (e.g., presence or absence of a phase transition, number and organization of pure states in the spin glass phase, etc.) — at least for very low temperature. While this remains an assumption, it is a common one in theoretical spin glass studies - so, for example, the thermodynamics of two extreme cases, the $\pm J$ and Gaussian distributions, are usually assumed to be the same [37].

All of the models discussed here have the desired properties at finite $\lambda$ (i.e., when $T>0$ ). At infinite $\lambda(T=0)$ they do not, and we make no claims as to whether any ground state properties of ordinary spin glasses can be inferred from these highly disordered models. But the interesting aspect of the analysis is that, while the thermodynamic properties at nonzero temperature cannot be directly solved for, the ground state properties of our models can, and the analysis in Sec. IV enables us to infer properties of realistic spin glass models at low but nonzero temperatures.

In general, the number of pure states for a given system remains the same or increases (at a phase transition point) as temperature decreases. Of course, this general tendency is violated at a first-order phase transition, due to phase coexistence. One well-known example of such a violation is the $q$-state Potts ferromagnet with $q$ sufficiently large (depending on $d$ ), which at $T_{c}$ has its paramagnetic pure phase coexisting with its $q$ ordered pure phases, while below $T_{c}$ the 
paramagnetic phase is unstable (for rigorous proofs, see [38 40]). However, it remains the case, for these and other systems with first-order transitions, that the number of pure phases above the transition is no larger than the number below. More interesting re-entrant behavior occurs in other systems - see, e.g., Ref. [41]. (A different type of re-entrant behavior occurs in some spin glasses in the temperature-concentration phase diagram [5], but this doesn't appear to violate the general rule of number of pure states not decreasing as temperature decreases.)

However, we are unaware of any natural examples where the number of ground states is smaller than the number of pure states at arbitrarily low temperature [42]. For the spin glass, we are looking at an even weaker claim - namely, that the number of pure states in the metastate does not decrease as temperature is lowered. That is, it is sufficient to consider only incongruent pure states, as discussed in Sec. III.

These considerations enable us to draw a set of interesting conclusions from our analysis of these models. The most natural, and obvious, one is that the number of ground states in the zerotemperature highly disordered metastate (e.g., with free or periodic boundary conditions) provides an upper bound to the number of pure states at very low temperature seen in realistic spin glass models. This ground state structure is known: the $T=0$ metastate of the highly disordered model is supported on a single pair of ground states below eight dimensions, and (uncountably) infinitely many above eight [15,17]. Our analysis therefore provides evidence in favor of the existence of no more than a single pair of pure states at low temperatures in realistic spin glass models below eight dimensions [43]. (This conclusion is consistent, of course, with there being only a single pure state, either paramagnetic or otherwise, at all nonzero temperatures in some or all of these dimensions.) Our analysis does not allow us to draw conclusions about what happens above eight dimensions.

There are two other logical possibilities, either of which would also be quite interesting. It could be that our general intuition about the behavior of the metastate as a function of temperature is violated here, so that the number of ground states is smaller than the number of pure states in some dimensions. In other words, in these models there might be a jump in the number of thermodynamic states, from larger to smaller, in the metastate at zero temperature. We cannot rule out this possibility, other to note that the discovery of a class of models in which this occurs raises the interesting possibility that it might occur elsewhere also.

Indeed, a jump presumably does occur in the ordinary $2 D$ EA Ising spin glass - but it goes in the other, more natural, direction, i.e. from smaller to larger as temperature is lowered to zero. The $2 D$ spin glass is believed to be paramagnetic at all nonzero temperatures, and has at least a pair of ground states at zero temperature. Our conclusion is that, while we cannot logically exclude the possibility of a lowering of the number of thermodynamic states as temperature goes to zero, it appears to be less likely than the number remaining the same or increasing.

We mention in passing a third possibility - that the number of pure states in a typical spin glass metastate does not behave in a continuous or even monotonic fashion at all as temperature is lowered [21]. This possibility presents a picture of the low-temperature spin glass phase far different from any that have appeared so far in the literature. An extreme version of this possibility (bearing some similarity to eigenvalue dependence on disorder realizations in low dimensional localization), which we present primarily for illustrative purposes, is as follows. At any (low) temperature not depending on the coupling realization $\mathcal{J}$, there would be no broken symmetry (and a unique infinite volume Gibbs state) for almost every $\mathcal{J}$, but nevertheless, if a typical $\mathcal{J}$ were picked first and then the temperature $T$ were varied, there would be a (countably infinite) 
dense set of temperatures, depending on $\mathcal{J}$, with broken symmetry pure phases for that $\mathcal{J}$ at those temperatures! However, because there is no evidence of such a picture to date, we do not pursue it further here.

Acknowledgments. This research was supported in part by NSF Grants DMS-98-02310 (CMN) and DMS-98-02153 (DLS). The authors thank Anton Bovier, Aernout van Enter, Christof Külske and Peter Young for useful discussions and constructive comments. 


\section{REFERENCES}

[1] C.M. Newman and D.L. Stein, Phys. Rev. Lett. 76, 515 (1996).

[2] C.M. Newman and D.L. Stein, Phys. Rev. E 55, 5194 (1997).

[3] C.M. Newman and D.L. Stein, Phys. Rev. E 57, 1356 (1998).

[4] M. Mézard, G. Parisi, and M.A. Virasoro, Spin Glass Theory and Beyond (World Scientific, Singapore, 1987).

[5] K. Binder and A.P. Young, Rev. Mod. Phys. 58, 801 (1986).

[6] E. Marinari, G. Parisi, F. Ricci-Tersenghi, J.J. Ruiz-Lorenzo, and F. Zuliani, J. Stat. Phys. 98, 973 (2000).

[7] W.L. McMillan, J. Phys. C 17, 3179 (1984).

[8] A.J. Bray and M.A. Moore, Phys. Rev. Lett. 58, 57 (1987).

[9] D.S. Fisher and D.A. Huse, Phys. Rev. Lett. 56, 1601 (1986).

[10] D.S. Fisher and D.A. Huse, J. Phys. A 20, L1005 (1987).

[11] D.S. Fisher and D.A. Huse, Phys. Rev. B 38, 386 (1988).

[12] C.M. Newman and D.L. Stein, Phys. Rev. B 46, 973 (1992).

[13] C.M. Newman and D.L. Stein, Phys. Rev. Lett. 76, 4821 (1996).

[14] S. Edwards and P.W. Anderson, J. Phys. F 5, 965 (1975).

[15] C.M. Newman and D.L. Stein, Phys. Rev. Lett. 72, 2286 (1994).

[16] J.R. Banavar, M. Cieplak, and A. Maritan, Phys. Rev. Lett. 72, 2320 (1994).

[17] C.M. Newman and D.L. Stein, J. Stat. Phys. 82, 1113 (1996).

[18] C.M. Newman and D.L. Stein, Phys. Rev. Lett. 84, 3966 (2000).

[19] A completely rigorous proof, though, has so far been obtained only for $d=2$.

[20] D.A. Huse and D.S. Fisher, J. Phys. A 20, L997 (1987).

[21] This should not be confused with the milder phenomenon of chaotic temperature dependence [8],9], in which the pure states themselves change chaotically with temperature but their number does not.

[22] O. Motrunich, S.-C. Mau, D.A. Huse, and D.S. Fisher, Phys. Rev. B 61, 1160 (2000).

[23] M. Aizenman and J. Wehr, Commun. Math. Phys. 130, 489 (1990).

[24] F. Krzakala and O.C. Martin, Phys. Rev. Lett. 85, 3013 (2000).

[25] M. Palassini and A.P. Young, Phys. Rev. Lett. 85, 3017 (2000).

[26] H.G. Katzgraber, M. Palassini, and A.P. Young, cond-mat/0007113, preprint (2000).

[27] E. Marinari and G. Parisi, cond-mat/0005047, preprint (2000).

[28] C.M. Newman and D.L. Stein, cond-mat/0010033, preprint (2000).

[29] R.L. Dobrushin, Theor. Prob. Appl. 17, 582 (1972).

[30] A.C.D. van Enter and J. Fröhlich, Commun. Math. Phys. 98, 425 (1985).

[31] A. Gandolfi, C.M. Newman and D.L. Stein, Commun. Math. Phys. 157, 371 (1993).

[32] Below four dimensions, quenched disorder is believed to result in domain wall wandering even at zero temperature. Domain wall wandering occurs also in uniform ferromagnets above the roughening temperature (which is zero in two dimensions and believed to be strictly below $T_{c}$ in three dimensions), but for a different reason - resulting from thermal fluctuations, rather than from quenched disorder.

[33] D.A. Huse and C.L. Henley, Phys. Rev. Lett. 54, 2708 (1985).

[34] M. Kardar, Phys. Rev. Lett. 55, 2923 (1985).

[35] A. Bovier and C. Külske, Rev. Math. Phys. 6, 413 (1994).

[36] A. Bovier and J. Fröhlich, J. Stat. Phys. 44, 347 (1986).

[37] Hence, the possibility of a transition at finite $\lambda_{c}$ from many pure states to a single pair, while logically possible, seems unlikely. The presence of such a transition would strongly suggest that the structure of 
a low-temperature spin glass phase could indeed be different for, say, the Gaussian and $\pm J$ coupling distributions. In this context, it is interesting to note that there is a mean-field Hopfield-type model in which the number of pure states is sensitive to the exact distribution of the couplings, but the mechanism there seems to not be relevant to our situation of EA spin glasses; see A. Bovier, B. Niederhauser, and A. van Enter, J. Stat. Phys. 95, 181 (1999).

[38] R. Kotecký and S.B. Shlosman, Commun. Math. Phys. 83, 493 (1982).

[39] D.H. Martirosian, Commun. Math. Phys. 105, 281 (1986).

[40] L. Laanait, A. Messager, and J. Ruiz, Commun. Math. Phys. 105, 527 (1986).

[41] R.H. Schonmann and N.I. Tanaka, Ann. Appl. Prob. 8, 234 (1998).

[42] There is though a handcrafted example of a lattice field theory with a single ground state but a sequence of temperatures tending to zero with coexisting phases; see E.A. Pecherski and S.B. Shlosman, Theor. Math. Phys. 70, 325 (1987).

[43] The possibility that dimension eight plays a special role in short-ranged spin glasses has been suggested previously, but for different reasons; see D.S. Fisher and H. Sompolinsky, Phys. Rev. Lett. 54, 1063 (1985). However, we emphasize that our claim is not that dimension eight necessarily plays a special role - only that the most likely conclusion of our analysis, of a single pair of low-temperature pure states, holds at least up to eight dimensions. 